\title{
The impact of disposal of fine grained sediments from maintenance dredging works on SPM concentration and fluid mud in and outside the harbor of Zeebrugge
}

\author{
Fettweis Michael', Matthias Baeye', Claudio Cardoso², Arvid Dujardin², Dries Van den Eynde', Thomas \\ Van Hoestenberghe ${ }^{2}$, Joris Vanlede ${ }^{3}$, Luc Van Poucke ${ }^{2}$, Carlos Velez $^{2}$ and Chantal Martens ${ }^{4}$ \\ 1 Operational Directorate Natural Environment, Royal Belgian Institute of Natural Sciences, \\ Gulledelle 100, 1200 Brussels, Belgium \\ E-mail: mfettweis@naturalsciences.be \\ 2 Antea Group, Buchtenstraat 9, 9051 Gent, Belgium \\ 3 Flanders Hydraulics Research, Department of Mobility and Public Works, Berchemlei 115, 2140 \\ Antwerp, Belgium \\ 4 Maritime Access Division, Department of Mobility and Public Works, Tavernierkaai 3, 2000 Antwerp, \\ Belgium
}

The amount of sediments to be dredged and disposed depends to a large part on the Suspended Particulate Matter (SPM) concentration. Tidal, meteorological, climatological and seasonal forcings have an influence on the horizontal and vertical distribution of the SPM in the water column and on the bed, and control the inflow of fine-grained sediments towards harbors and navigation channels. About 3 million tons (dry matter) per year of mainly fine-grained sediments is dredged in the port of Zeebrugge and is disposed on a nearby disposal site. The disposed sediments are quickly resuspended and transported away from the site.

The hypothesis is that a significant part of the disposed sediments recirculates back to the dredging places and that a relocation of the disposal site to another location at equal distance to the dredging area would reduce this recirculation. In order to validate the hypothesis a one year field study was set up in 2013-2014. During one month the dredged material was disposed at a new site. Variations in SPM concentration were related to tides, storms, seasonal changes and human impacts. In the highturbidity Belgian near shore area the natural forcings are responsible for the major variability in the SPM concentration signal, while disposal has only a smaller influence. The conclusion from the measurements is that the SPM concentration decreases after relocation of the disposal site, but indicate stronger (first half of field experiment) or weaker (second half of field experiment) effects that are, however, supported by the environmental conditions. The results of the field study may have consequences on the management of disposal operations as the effectiveness of the disposal site depends on environmental conditions, which are inherently associated with chaotic behavior.

Keywords: dredging and disposal; human impact; SPM concentration 